\title{
Minireview
}

\section{Retention of glycosyltransferases in the Golgi apparatus}

\author{
Ewa Jaśkiewicz
}

\begin{abstract}
Department of Immunochemistry, Ludwik Hirszfeld Institute of Immunology and Experimental Therapy, Polish Academy of Sciences, Czerska 12, 53-114 Wroctaw, Poland
\end{abstract}

Received: 19 November, 1996; accepted: 22 April, 1997

Key words: Golgi apparatus, glycosyltransferases, retention

\begin{abstract}
A number of Golgi glycosyltransferases has been cloned to date. They all are membrane proteins and share the same type II topology, but they do not possess an obvious sequence homology which would suggest a common Golgi retention signal. However, it was shown that the membrane-spanning domain and its flanking regions contain necessary and sufficient information for Golgi retention of these enzymes. Currently, two mutually complementary models have been proposed to explain the mechanism of Golgi retention of glycosyltransferases mediated by their transmembrane domain. The first model postulates the retention through oligomerization, which prevents enzymes from entering the transport vesicles. The second suggests that retention depends on the length of a membrane-spanning domain and thickness of the membrane along the Golgi complex. It has to be pointed out that neither the oligomerization nor the membrane thickness model alone can answer all questions and further work is still needed to elucidate the retention process of Golgi proteins.
\end{abstract}

\section{RETENTION AND RETRIEVAL MECHANISMS TO ACHIEVE ORGANELLE RESIDENCE}

The eucaryotic secretory pathway consists of a series of membraneous organelles defined by their characteristic morphology and the presence of resident luminal and membrane proteins. Nascent secretory proteins, following their synthesis on endoplasmic reticulum (ER), are translocated into the lumen of the ER, where they are core-glycosy- lated, correctly folded and oligomerized, before they leave ER. These proteins must then be distributed to their correct destinations, including the subcellular compartments and the plasma membrane, or secreted outside the cell. All intracellular transport between organelles is mediated by membrane vesicles, with a bulk flow of lipids.

Recent advances in the studies on the structure and function of coated transport vesicles have revealed not only their role as carriers but also their direct involvement in the proc-

Presented at the V Symposium on Glycoconjugates, XXXII Meeting of the Polish Biochemical Society, Kraków (Poland), September 17-20, 1996

Abbreviations: COS cells, African green monkey cells; CHO cells, Chinese hamster ovary cells; ER, endoplasmic reticulum; NAGT I, $N$-acetylglucosaminyltransferase I; GT, $\beta$-1,4-galactosyltransferase; ST, $\alpha$-2,6-sialyltransferase; MDCK, Madin-Darby canine kidney cell line. 
ess of protein sorting [1]. It has been proposed that there are three potential possibilities for a protein interaction with a transport vesicle:

$\downarrow 1$. The protein possesses a sorting signal termed a "transport signal" that binds to a coat protein of the vesicle, directly or indirectly, by a specific transmembrane receptor. The protein becomes concentrated in a vesicle during budding and is transported to an organelle of its destination defined by the second type of sorting signal termed a "retention signal".

$\downarrow 2$. The protein is restricted from entering a budding vesicle by a "retention signal", which is organelle specific. It is thought that the retention my occur by formation of aggregates of retained proteins that do not enter a transport vesicle, because of their size or interaction with a Golgi matrix.

$\downarrow 3$. The protein which does not contain any signal diffuses into a vesicle and then is transported out by default at bulk flow rate.

In summary, each protein with a given destination should have at least two independent sorting signals specifying its intracellular localization. The process of specific localization of each protein would be thus an effect of cooperation of two mechanisms: retention and retrieval [2]. The retention process would ensure a compartmental localization by preventing the entrance of a protein to transport vesicles of the anterograde pathway. The retrieval mechanism would operate only when the protein had left the compartment of its residence and would ensure its retrograde transport based on a specific receptor. It is understood that the protein residency is the result of the balance between the strength of the retention and the retrieval mechanism. The less efficient retention has to be compensated by retrieval to ensure compartments to retain their own distinct sets of proteins.

Examples of the known transport signals are summarized in Table 1. All of them operate in pre- or post-Golgi compartments. It should be emphasized that no signal responsible for anterograde transport of proteins from ER to the Golgi complex has been identified so far. It seems that Golgi residents have to possess only a retention signal to maintain them in a specific Golgi compartment.

\section{THE MEMBRANE-SPANNING DOMAIN AND ITS FLANKING SEQUENCES IS A SIGNAL FOR RETENTION OF GLYCOSYLTRANSFERASES}

Most of Golgi proteins cloned to date are glycosyltransferases involved in the elongation of oligosaccharides of newly synthesized glycoproteins and glycolipids [3]. They all are membrane proteins, which share a common type II topology (carboxyl terminus in the lumen), with a short cytoplasmic tail, single noncleavable transmembrane domain connected via a "stem" region with the luminal catalytic domain (Fig. 1). There is, however, no amino-acid sequence similarity among these Golgi proteins. Therefore it is difficult to predict a retention mechanism based on a specific receptor. In addition, Golgi retention of glycosyltransferases can not be saturated by overexpression. Thus, this does not lead to cell-surface expression, but instead, enzymes are returned to $\mathrm{ER}[4,5]$.

The Golgi retention of three glycosyltransferases: $N$-acetylglucosaminyltransferase I (NAGT I), ק-1,4-galactosyltransferase (GT) and $\alpha$-2,6-sialyltransferase (ST) have been extensively studied. The specific Golgi localization of these enzymes was defined using subcellular fractionation of Golgi membranes and electron microscopy. Several groups have localized NAGT I predominantly in the medial-Golgi cisternae, GT in the trans-Golgi cisternae and ST in the transcisternae of the Golgi and trans-Golgi network of different cell types. To define the signal for retention of these enzymes in the Golgi apparatus generally two approaches were used. First, the Golgi residents were expressed in a suitable cell line and their retention was disrupted by removal or replacement of polypeptide chain fragments. Further, these putative sequences were fused to the non-Golgi reporter proteins and the localization of the chimeric proteins was monitored using immunofluorescence or immunoelectron microscopy. 
Table 1. Examples of known transport signals.

According to Rothman \& Wieland [1].

\begin{tabular}{|c|c|c|}
\hline Signal & Location in protein & Destination \\
\hline KDEL* & $\mathrm{COOH}$-terminus, luminal & $\begin{array}{l}\text { Retrieval of proteins from Golgi to } \\
\text { ER }\end{array}$ \\
\hline KKXX & COOH-terminus, in cytoplasm & $\begin{array}{l}\text { Retrieval of membrane proteins } \\
\text { from Golgi to ER }\end{array}$ \\
\hline XXRR & $\mathrm{NH}_{2}$-terminus, in cytoplasm & $\begin{array}{l}\text { Retrieval of membrane proteins } \\
\text { from Golgi to ER }\end{array}$ \\
\hline Mannose 6-phosphate & Asn-linked saccharides, luminal & $\begin{array}{l}\text { Transport from Golgi to } \\
\text { endosomes/lysosomes }\end{array}$ \\
\hline Tyrosine-rich and dileucine & Cytoplasmic domain & $\begin{array}{l}\text { Transport from Golgi to } \\
\text { endosomes/lysosomes }\end{array}$ \\
\hline YQRL (and similar) & Cytoplasmic domain & Transport from cell surface to Golgi \\
\hline NPXY ( and similar) & Cytoplasmic domain & $\begin{array}{l}\text { Transport from cell surface to } \\
\text { endosomes }\end{array}$ \\
\hline GPI anchor & COOH-terminus, luminal & $\begin{array}{l}\text { Transport from Golgi to apical cell } \\
\text { surface in polarized cells }\end{array}$ \\
\hline
\end{tabular}

*K, Lys; D, Asp; E, Glu; L, Leu; R, Arg; Y, Tyr; Q, Gln; N, Asn; P. Pro; X, any amino acid. GPI, glycosylphosphatidylinositol.

The retention of the medial-Golgi enzyme NAGT I was examined by Tang et al. [6] in MDCK cells. Different N-terminal portions of this enzyme were attached to the ectodomain of dipeptydyl peptidase IV, which is type II membrane surface protein. The authors have found that the presence of the transmembrane domain of NAGT I is important to localize the fusion proteins to the Golgi apparatus, however, a significant amount of chimeric proteins was still found at plasma membrane. The retention was more efficient when the cytoplasmic tail and a part of "stem" region were also present. The contribution of all three parts of molecule to the Golgi retention of this enzyme was confirmed

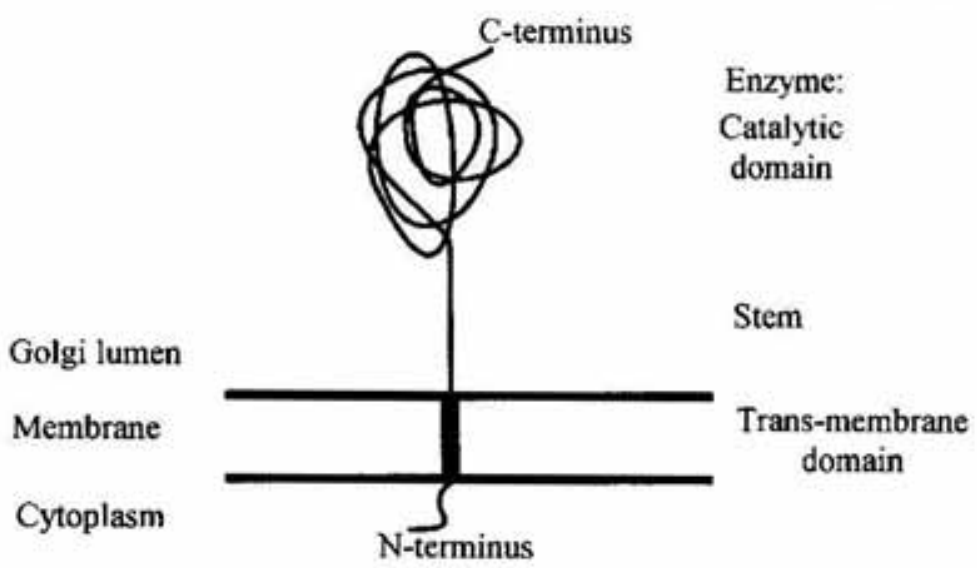

by Burke et al. [7] in murine L cells, using chimeras containing domains from NAGT I, ovalbumin and the transferrin receptor. Recent results of Nilsson et al. [8] suggest, however, that the transmembrane domain of NAGT I is not necessary for its Golgi retention, but the "stem" sequences alone are necessary and sufficient for retention of the enzyme in HeLa cells.

The same group have examined the localization signal for the trans-Golgi enzyme GT in HeLa cells [4]. Chimeric constructs possessing the cytoplasmic tail, transmembrane domain and a few luminal amino acids from GT fused to the luminal domain of invariant chain (Iip33, a plasma membrane and en-

Enzyme:

Catalytic

domain

Figure 1. Domain structure of a glycosyltransferase enzyme. 
dosomal protein) were retained in the transGolgi membrane. The efficient Golgi retention required also the cytoplasmic tail. Studies of Masibay et al. [9] in COS cells have also confirmed that the GT transmembrane domain is necessary and sufficient for its Golgi retention and pointed out that the length of the hydrophobic region of transmembrane domain is an important factor for Golgi retention of GT. Similarly, Aoki et al. [10], using deletion, replacement and point mutants, have confirmed the role of membranespanning domain in GT retention in COS cells. They have shown that the cytoplasmic half of the transmembrane domain of GT, particularly Cys-29 and His-32, contribute to the Golgi retention signal. It was proposed that retention of GT could be an effect of the homodimers formation, which is dependent on cysteine and histidine residues within the transmembrane domain [11].

Colley and colleagues [12, 13] have examined the retention of another trans-Golgi enzyme: ST in COS cells. Studies with deletion and replacement mutants and chimeric proteins suggested that the ST transmembrane domain is not sufficient for its Golgi retention, and it is rather cytoplasmic and luminal flanking sequences that are more important fragments of the ST Golgi retention signal. This was also confirmed by Munro [5] who has shown that a reporter construct with the ST "stem" and cytoplasmic tail, but with 17 leucine residues replacing the ST transmembrane domain, was still retained in the Golgi apparatus. But a similar construct possessing 23 leucine residues was found at the cell surface. It seems that in case of ST the correctly spaced flanking sequences are important for its Golgi retention [5, 12-14]. Besides, the "stem" sequences alone can act as an independent Golgi retention signal [12, 15].

From the studies of several groups it can be concluded that the membrane spanning domains of glycosyltransferases are necessary for their Golgi retention, but their cytoplasmic and/or luminal sequences play often accessory or even independent roles in this process. The divergent data obtained by different researchers could be partially explained by the use of different cell types for

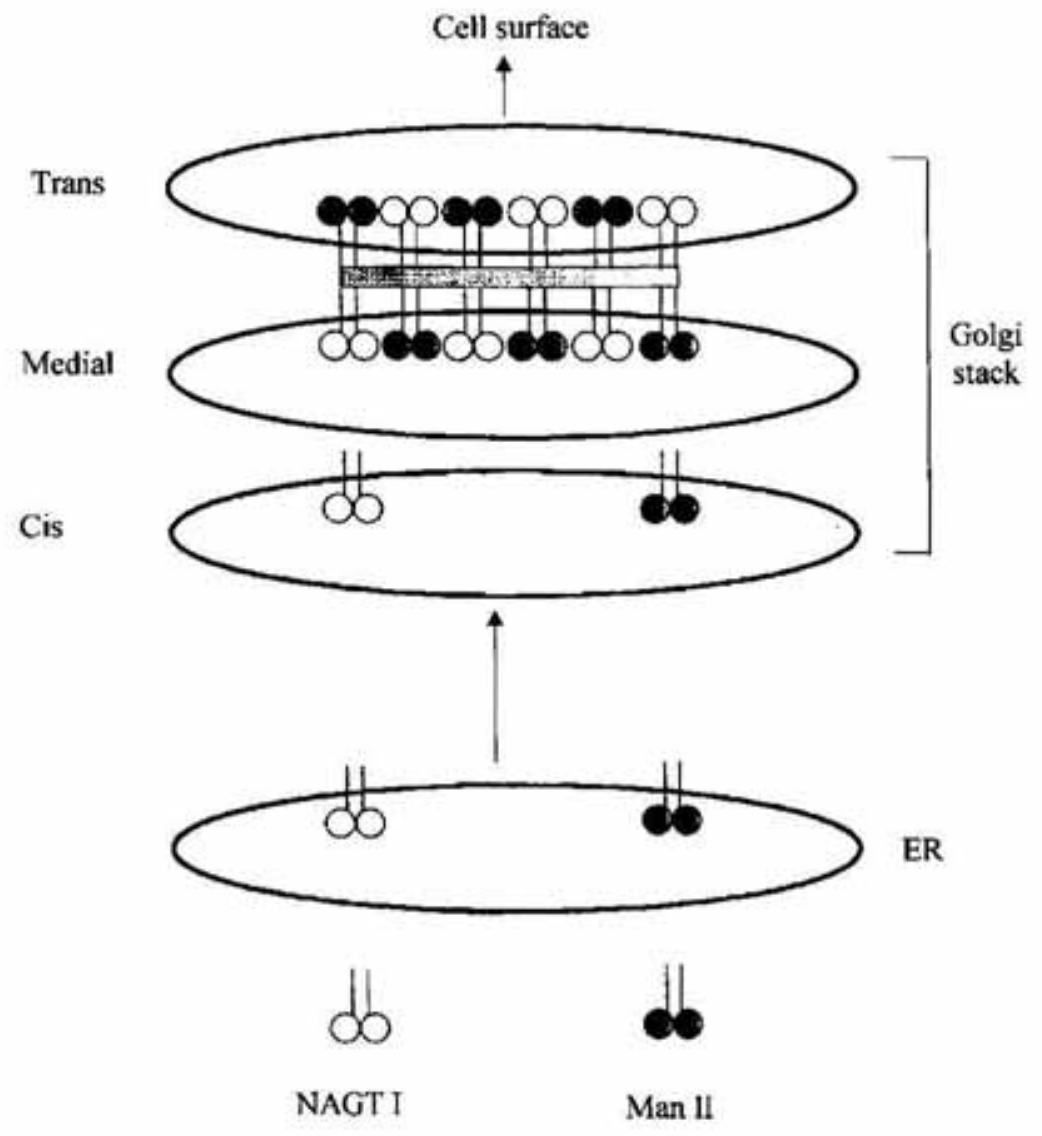

Figure 2. Model of "kin recognition" of the Golgi glycosyltransferases.

The homo-dimers of $\mathrm{N}$-acetylglucosaminyltransferase and mannosidase II (ManII) are transported along the secretory pathway until they interact to form "kin" oligomers. The cytoplasmic tails of these enzymes are bound by a Golgi matrix. ER, endoplasmic reticulum; cis, medial and trans Golgi apparatus. 
protein expression, variety of reporter proteins used in chimeric constructs, different levels of protein expression and wide range of sensitivity of detection methods [15].

\section{TWO MODELS OF THE GOLGI RETENTION: THROUGH OLIGOMERIZATION AND MEMBRANE THICKNESS}

Although the mechanism underlying the transmembrane domain-mediated retention of glycosyltransferases has not yet been resolved, two mutually complementary models have been proposed. The first model postulates the retention through oligomerization of molecules. The second model proposes that retention depends on fitting the length of membrane-spanning domain to the membrane thickness.

Swift \& Machamer [16] basing on their studies on the coronavirus M (E1) glycoprotein, have first proposed, that Golgi proteins could form homo-oligomers which do not enter the forward moving transport vesicles and are thus retained. This model requires that aggregation is induced at a particular point during Golgi transit. Transport could be prevented by size and/or interactions with Golgi matrix [17].

Nilsson et al. [18] have postulated an extended model of oligomerization called "kin recognition". They had shown that two medial-Golgi enzymes NAGT I and mannosidase II interact via their transmembrane domains to form large hetero-oligomers (Fig. 2 ). These oligomers could attach to the matrix with their cytoplasmic domains, which prevents a forward movement of the enzymes [19]. The results supporting the role of cytoskeletal proteins in the Golgi retention were obtained by Yamaguchi \& Fukuda [11]. They have found that GT ( $\alpha$ trans-Golgi enzyme) forms homo-oligomers, which are stabilized in the Golgi by their direct or indirect association with tubulins. Nilsson et al. [18] proposed that the "kin recognition" model is universal, suggesting that, in general, the enzymes residing in the same Golgi compartment form hetero-oligomers and are thus retained. However, this supposition has not been confirmed by Munro in his studies on two trans-Golgi enzymes: ST and GT [14]. Since no evidence could be obtained for specific interactions or "kin recognition" between ST and other trans-Golgi proteins. Moreover, it was shown that the previously described "kin recognition" between the medial-Golgi enzymes (NAGT I and mannosidase II) involved the luminal portions of these proteins rather than their transmembrane domains [14].

This and previous observations [5, 9, 13], have led Bretcher \& Munro [20] to propose that the length of the membrane spanning domain might be an important factor for the Golgi retention. This model is based on two observations: first, that transmembrane domains of Golgi proteins are generally on average by five amino acids shorter, than those of plasma membrane proteins [21], second, it seems that membrane thickness is increasing along the secretory pathway, due to a cholesterol concentration gradient [22]. The retention of Golgi enzymes across the Golgi complex would be thus an effect of the length of their membrane-spanning domains fitting the thickness of the adjacent bilayer. The position of a retained molecule would be then energetically most favorable.

It seems that neither mechanism alone is completely satisfactory and both mechanisms might operate for the efficient retention of Golgi glycosyltransferases. Current data support this combined model [15]. The common feature of both models is that once a protein reaches its correct compartment within the Golgi apparatus it is excluded from forward movement and retained. We realize now however, that the retention is not fully restrictive, since localization of several enzymes is overlapping within the Golgi compartments with the maximum concentration in one of them [23]. Indeed, some mechanisms that have been ruled out before, such as recycling, have also to be considered. The most recent studies on NAGT I in ldID cells [24] and yeast mannosidase Och 1p [25] provided evidence for the movement of both enzymes to distal compartments of the Golgi stack, followed by retrieval to the compartment of destination. 


\section{CONCLUSIONS}

The results of the recent studies on the Golgi retention of three glycosyltransferases: $\mathrm{N}$-acetylglucosaminyltransferase I, $\beta$-1,4-galactosyltransferase and $\alpha$-2,6-sialyltransferase, lead to the following major conclusions:

$\downarrow 1$. The membrane-spanning domain of GT seems to be necessary and sufficient for its Golgi retention. In the case of NAGT I and $\mathrm{ST}$ their retention require the transmembrane domain together with its

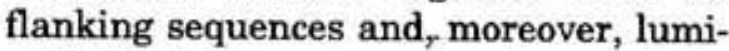
nal "stem" sequences may act as an independent retention signal.

$\downarrow 2$. The Golgi retention process of glycosyltransferases seems to be an effect of cooperation of several mechanisms including: homo- and hetero-oligomerization, association with cellular matrix and interaction with a lipid bilayer; however, involvement of some additional mechanisms can not be excluded.

\section{REFERENCES}

1. Rothman, J.E. \& Wieland, F.T. (1996) Protein sorting by transport vesicles. Science $\mathbf{2 7 2}$, 227-234.

2. Nilsson, T. \& Warren, G. (1994) Retention and retrieval in the endoplasmic reticulum and the Golgi apparatus. Curr. Opin. Cell Biol. 6, 517-521.

3. Field, M.C. \& Wainwright, L.J. (1995) Molecular cloning of eucaryotic glycoprotein and glycolipid glycosyltransferases: A survey. Gly. cobiology 5, 463-472.

4. Nilsson, T., Lucocq, J.M., Mackay, D. \& Warren, G. (1991) The membrane spanning domain of $\beta$-1,4-galactosyltransferase specifies trans-Golgi localization. EMBO J. 10, 35673575.

5. Munro, S. (1991) Sequences within and adjacent to the transmembrane segment of $\alpha-2,6$ sialyltransferase specify Golgi retention. EMBO J. 10, 3577-3588.

6. Tang, B.L., Wong, S.H., Low, S.H. \& Hong, W. (1992) The transmembrane domain of $N$-glu- cosaminyltransferase I contains a Golgi retention signal. J. Biol. Chem. 267, 1012210126.

7. Burke, J., Pettit, J.M., Humpris, D. \& Gleeson, P.A. (1994) Medial-Golgi retention of $\mathrm{N}$-acetylglucosaminyltransferase I. J. Biol. Chem. 269, 12049-12059.

8. Nilsson, T., Rabouille, C., Hui, N., Watson, R. \& Warren, G. (1996) The role of the membrane-spanning domain and stalk region of $\mathrm{N}$-acetylglucosaminyltransferase $\mathrm{I}$ in retention, kin recognition and structural maintenance of the Golgi apparatus in HeLa cells. $J$. Cell Sci. 109, 1975-1989.

9. Masibay, A.S., Balaji, P.V., Boeggeman, E.E. \& Qasba, P.K. (1993) Mutational analysis of the Golgi retention signal of bovine $\beta-1,4$ galactosyltransferase. J. Biol. Chem. 268, 9908-9916.

10. Aoki, D., Lee, N., Yamaguchi, N., Dubois, C. \& Fukuda, M.N. (1992) Golgi retention of a trans-Golgi membrane protein, galactosyltransferase, requires cysteine and histidine residues within the membrane anchoring domain. Proc. Natl. Acad. Sci. U.S.A. 89, 43194323.

11. Yamaguchi, N. \& Fukuda, M.N. (1995) Golgi retention mechanism of $\beta$-1,4-galactosyltransferase. J. Biol. Chem. 270, 12170-12176.

12. Colley, K.J., Lee, E.U. \& Paulson, J.C. (1992) The signal anchor and stem regions of the $\beta$-galactoside $\alpha$-2,6-sialyltransferase may each act to localize the enzyme to the Golgi apparatus. J. Biol. Chem. 267, 7784-7793.

13. Dahdal, R.Y. \& Colley, K.J. (1993) Specific sequences in the signal anchor of the $\beta$-galactoside $\alpha^{*}(2,6)$-sialyltransferase are not essential for Golgi localization. J. Biol. Chem. 268, 26310-26319.

14. Munro, S. (1995) An investigation of the role of transmembrane domains in Golgi protein retention. EMBO J. 14, 4695-4704.

15. Colley, K.J. (1997) Golgi localization of glycosyltransferases: More questions than answers. Glycobiology 7, 1-13.

16. Swift, A.M. \& Machamer, C.E. (1991) A Golgi retention signal in a membrane spanning do- 
main of a coronavirus E1 glycoprotein. $J$. Cell Biol. 115, 19-30.

17. Machamer, C.E. (1993) Targeting and retention of Golgi membrane proteins. Curr. Opin. Cell Biol. 5, 606-612.

18. Nilsson, T., Hoe, M.H., Slusarewicz, P., Rabouille, C., Watson, R., Hunte, F., Watzele, G., Berger, E.G. \& Warren, G. (1994) Kin recognition between medial-Golgi enzymes in HeLa cells. EMBO J. 13, 562-574.

19. Slusarewicz, P., Nilsson, T., Hui, N., Watson, R. \& Warren, G. (1994) Isolation of a matrix that binds medial-Golgi enzymes. J. Cell Biol. 124, $405-413$.

20. Bretcher, M.S. \& Munro, S. (1993) Cholesterol and the Golgi apparatus. Science 261, 12801281.

21. Munro, S. (1995) A comparison of the transmembrane domains of Golgi and plasma membrane proteins. Biochem. Soc. Trans. 23, 527-530.
22. Orci, L., Montesano, R., Meda, P., Malaise-Lagae, F., Brown, D., Perrelet, A. \& Vassalli, P. (1981) Heterogenous distribution of Filipincholesterol complexes across the cisternae of the Golgi apparatus. Proc. Natl. Acad. Sci. U.S.A. 78, 293-297.

23. Nilsson, T., Paypayert, M., Hoe, M.H., Slusarewicz, P., Berger, E.G. \& Warren, G. (1993) Overlaping distribution of two glycosyltransferases in the Golgi apparatus of HeLa cells. J. Cell Biol. 120, 5-13.

24. Hoe, M.H., Slusarewicz, P., Misteli, T., Watson, R. \& Warren, G. (1995) Evidence for recycling of the resident medial/trans-Golgi enzyme, $\mathrm{N}$-acetylglucosaminyltransferase $\mathbf{I}$, in IdID cells. J. Biol. Chem. 270, 2505735063 .

25. Harris, S.L. \& Waters, M.G. (1996) Localization of a yeast early Golgi mannosyltransferase, Och $1 \mathrm{p}$, involves retrograde transport. J. Cell Biol. 132, 985-998. 